Check for updates

Cite this as: $B M J 2021 ; 372: n 615$ http://dx.doi.org/10.1136/bmi.n615 Published: 10 March 2021

\section{Update to living systematic review on covid-19 in pregnancy}

This living systematic review on coronavirus disease 2019 (covid-19) by Allotey and colleagues (BMJ 2020;370:m3320) has been updated. For the latest update, visit doi:. This update includes 192 studies (64 676 pregnant and recently pregnant women), 115 of which were new additions to the review. The prevalence of covid-19 in pregnant and recently pregnant women remained unchanged between this update and the original version of the living systematic review. Pregnant women continue to be at increased risk of severe covid-19. In addition to high body mass index and advancing maternal age, evidence suggests that non-white ethnic origin might also be a risk factor for severe covid-19. More data are needed to robustly assess the association between pregnancy specific risk factors and covid-19 related outcomes. 